\title{
Comportamento da carga de treinamento, recuperação e bem-estar em atletas profissionais de voleibol em semanas com e sem jogos
}

Behavior of the training load, recovery and well-being in volleyball professional athletes in weeks with and without matches

\section{Fernanda Martins Brandão}

Universidade Federal de Juiz de Fora - Faculdade de Educação Física e Desportos. Grupo de Estudos Controle da Carga de Treino. , Brasil

fernandamabrandao@gmail.com

\section{Vinícius Figueirôa da Cunba}

Universidade Federal de Juiz de Fora - Faculdade de Educação Física e Desportos. Grupo de Estudos Controle da Carga de Treino. , Brasil

viniciusfigueiroa@hotmail.com

\section{Thiago Ferreira Timoteo}

Universidade Federal de Juiz de Fora - Faculdade de Educação Física e Desportos. Grupo de Estudos Controle da Carga de Treino. , Brasil

thiagoftimoteo@gmail.com

\section{Thiago Seixas Duarte}

Universidade Federal de Juiz de Fora - Faculdade de Educação Física e Desportos, Brasil tseixas.ef@gmail.com

\section{Bernardo Miloski Dias}

Universidade de São Paulo - Escola de Educação Física e Esporte, Brasil

bernardomiloski@yahoo.com.br

\section{Danilo Reis Coimbra}

Universidade do Estado de Santa Catarina - Centro de Educação Física e Desportos, Brasil

daniloreiscoimbra@yahoo.com.br

\section{Renato Miranda}

Faculdade de Educação Física da Universidade Federal de Juiz de Fora, Brasil

mrena@terra.com.br

\section{Mauricio Gattás Bara Filho}

Faculdade de Educação Fisica da Universidade Federal de Juiz de Fora. Grupo de Estudos Controle da Carga de Treino., Brasil

mgbara1973@gmail.com 


\section{Resumo:}

O objetivo deste estudo foi comparar a carga interna de treinamento, o estado de recuperação e o bem-estar em atletas profissionais de Voleibol de acordo com a frequência semanal de jogos. A recuperação foi quantificada pela Escala de TQR, a carga pela PSE da sessão e o bem-estar pela escala de QBE. Para comparar os valores da Carga de Treinamento Semanal Total, o delta da Qualidade Total de Recuperação $(\Delta \mathrm{TQR})$ e o delta da Qualidade de bem-estar $(\triangle \mathrm{QBE})$ foi aplicado o teste ANOVA para medidas repetidas. Não foram encontradas diferenças significativas ao comparar a carga de treinamento com a frequência de jogos semanais, porém o tamanho do efeito foi muito elevado quando se comparou a diferença entre um e dois jogos semanais, assim como de nenhum a dois jogos na semana. Houve diferença entre o $\triangle T Q R$ entre as semanas sem jogos e com dois jogos assim como entre as semanas com um e dois jogos. Já o $\Delta \mathrm{QBE}$ foi diferente para as semanas que não havia a realização de jogos e aquelas com jogos. Concluise que, mesmo que não haja uma variação das cargas com diferentes frequências semanais de jogos, a recuperação assim como o bem-estar dos atletas foi alterada.

PalaVras-Chave: Voleibol, Carga de treinamento, Recuperação, Bem-estar.

\section{Abstract:}

The aim of this study was to compare the internal training load, recovery state and the well-being of volleyball professional athletes, according to the number of matches during the week. The recovery was quantified with TQR Scale, the training load with sRPE and the well-being with QBE Scale. The ANOVA was applied for repeated measures in order to compare the values of Total weekly training load, the delta of Total Quality Recovery $(\Delta \mathrm{TQR})$ and the delta of the quality of well-being $(\Delta \mathrm{QBE})$,. There was no significant difference among the training load and the frequency of weekly games, but the effect size was very high when it was compared to the difference between one and two weekly games, as well as any and two games in a week. There were differences on the $\triangle \mathrm{TQR}$ between weeks without games and with two games, as well as weeks with one and two matches. The $\Delta \mathrm{QBE}$ was different for weeks in which there was no game and those with matches, regardless of the frequency. It was concluded that, even if there is no variation of loads in different weekly frequency of matches, the athletes' recovery and well-being change.

KEYWORDS: Volleyball, Training Load, Recovery, Well-being.

\section{INTRODUÇÃO}

O treinamento esportivo é uma atividade sistematicamente organizada que através da sobrecarga visa proporcionar adequadas adaptações aos atletas, por meio de alterações morfológicas, metabólicas e psicológicas, com o objetivo de melhorar o desempenho competitivo (Impellizzeri, Rampinini, Coutts, Sassi, \& Marcora, 2004).

A partir disso, como forma de minimizar os efeitos negativos do treinamento (overreaching não-funcional, lesões ou doenças) e potencializar o desempenho dos atletas, é necessário que a comissão técnica realize um monitoramento preciso das cargas de treinamento (CT) (Bourdon \& Cardinale, 2017). De acordo com um modelo proposto por Impellizzeri et al. (2004) as CT são categorizadas em carga externa de treinamento (CET) e carga interna de treinamento (CIT). A CET é definida como uma medida objetiva sendo referente à carga prescrita pelo treinador associada à volume e intensidade. Já a CIT é determinada pela resposta ao estresse psicofisiológico imposto aos atletas, de maneira individual, através das sessões de treinamento planejadas ou competições.

No entanto, para que ocorra uma melhora do desempenho não se deve basear somente nas CT, já que o restabelecimento do estado homeostático dos atletas através de uma recuperação adequada também está intimamente ligado com o desempenho (Kenttä \& Hassmén, 1998). Assim, em um processo de treinamento ideal, é necessário haver um equilíbrio entre os estímulos estressores do treinamento com as estratégias de recuperação para que os atletas consigam apresentar e manter um saldo de treinamento e recuperação positivos durante todo período e consequentemente atinjam um bom desempenho, principalmente durante o período competitivo (Kellmann, 2010; McLean, Coutts, Kelly, McGuigan, \& Cormack, 2010).

Vários são os métodos descritos na literatura para realizar o monitoramento das CT (Borresen \& Lambert, 2009) e do estado de recuperação. Atualmente, a percepção subjetiva do esforço (PSE) da sessão (Foster, 1998; Foster et al., 2001) vem se destacando como um método confiável para avaliar a magnitude da CIT. Do mesmo modo, a escala de Qualidade Total de Recuperação (TQR) vem sendo apontada como um método 
simples e eficaz para o monitoramento da recuperação (Brink, Nederhof, Visscher, Schmikli, \& Lemmink, 2010). Em relação à recuperação, também é importante observar as questões que interferem nas condições de bem-estar dos atletas. Nesse sentido, um estudo realizado no futebol americano universitário concluiu que o monitoramento da CIT em conjunto com medidas subjetivas de bem-estar pode auxiliar os treinadores a antecipar com mais precisão o risco de um atleta apresentar alguma má adaptação ao treinamento (Govus, Coutts, Duffield, Murray, \& Fullagar, 2018). A partir disso, os métodos de natureza subjetiva estão em evidência pela sua simplicidade, baixo custo, por não serem invasivos e pela sua melhor aplicabilidade, já que seus resultados são interpretados com maior rapidez quando comparados a métodos objetivos, por exemplo (Saw, Main, \& Gastin, 2016).

Dentro deste contexto, o voleibol é um esporte coletivo com característica intermitente, sendo suas principais ações curtas e de alta intensidade, intercaladas por períodos de baixa intensidade (Sheppard, Gabbett, \& Stanganelli, 2009). O monitoramento da CIT em esportes coletivos, como o voleibol, é fundamental, já que ocorrem diferenças nas respostas individuais para a mesma CET planejada (Manzi et al., 2010). Além disso, o voleibol apresenta uma particularidade de ser um esporte em que não utiliza o tempo como marcação para determinar o fim de uma partida, e tal fato pode ser que gere maior dificuldade no monitoramento das cargas ao longo do período competitivo, uma vez que a comissão técnica não saberá quantos sets serão disputados em uma partida e o tempo de duração total da mesma.

A característica da semana também pode influenciar o comportamento das CT e do estado de recuperação. Um estudo realizado no basquete por Manzi et al. (2010) indicou que semanas sem jogos tendem a apresentar maior CIT quando comparada a semana com jogos. Este fato pode ser explicado devido à presença de um jogo ao fim da semana e, portanto, a CT não deveria ser intensificada, o que poderia levar prejuízo no desempenho dos atletas durante a competição.

Neste sentido, observa-se uma lacuna no conhecimento sobre a relação carga de treinamento e recuperação em semanas com e sem a presença de jogos em diferentes modalidades, incluindo o voleibol. Sendo assim, pesquisar o monitoramento adequado das variáveis de recuperação e bem-estar torna-se importante no esporte competitivo como meio de prevenir ou minimizar os efeitos negativos das CT. A partir do exposto, o objetivo do presente estudo foi comparar a CIT, o estado de recuperação e o bem-estar de atletas profissionais de voleibol e semanas com e sem jogos.

\section{Metodologia}

\section{Sujeitos:}

A amostra foi composta por 14 jogadores de voleibol (idade de 26,7 $\pm 5,5$ anos; massa corporal 95,8 \pm 8,2 kg; estatura 1,97 $\pm 7,9 \mathrm{~cm}$ ) profissionais, do sexo masculino, participantes de uma equipe de nível nacional que disputou a Superliga, principal competição do país na modalidade. Todos os participantes, após receberem as explicações sobre os objetivos e procedimentos do estudo, assinaram um termo de consentimento livre e esclarecido, aprovado pelo Comitê de Ética e Pesquisa da instituição de origem dos pesquisadores (N. ${ }^{\circ}$ 771/758). Os jogadores estavam amplamente familiarizados com os métodos utilizados na pesquisa, já que estes eram utilizados habitualmente pela comissão técnica da equipe investigada.

\section{Procedimentos:}

Foram monitoradas 33 semanas da temporada e estas foram divididas de acordo com a frequência de jogos semanais, resultando em: 8 semanas sem jogos, 10 semanas com a realização de um jogo e 11 semanas com a realização de dois jogos. Houve ainda uma semana de um torneio curto que contou com 5 jogos. 
Durante estas semanas foram coletados dados referentes à CIT, recuperação e bem-estar. Para cada semana, as cargas de treinamento de todas as sessões foram somadas, resultando assim na carga de treinamento semanal total (CTST). Os escores de bem-estar e recuperação foram obtidos sempre nos primeiros e últimos dias de treinamento da semana, sendo estes dados coletados antes dos treinos, enquanto a PSE de sessão foi coletada diariamente após os treinos.

\section{Instrumentos:}

\section{Percep̧̧ão Subjetiva do Esforço da sessão (PSE da Sessão)}

Para monitorar a CIT foi utilizado o método da PSE da sessão (Foster et al., 2001). Este método consiste na multiplicação do escore da PSE pela duração total da sessão expressa em minutos, sendo expressa em unidades arbitrárias. Assim, após 30 minutos do término de cada sessão de treinamento os atletas responderam a seguinte questão "Como foi a sua sessão de treino hoje?". Em seguida, os atletas apontaram sua resposta na escala (Quadro 1), sendo esta referente à sessão de treinamento como um todo. Assim, o valor máximo (10) deveria ser comparado ao maior esforço físico realizado pelo atleta e o valor mínimo é a condição de repouso absoluto (0).

\section{QUADRO 1}

Escala de PSE de 10 pontos adaptada por Foster et al. (2001)

\begin{tabular}{ll}
\hline 0 & Repouso \\
1 & Muito, muito leve \\
2 & Leve \\
3 & Médio \\
4 & Um pouco pesado \\
5 & Pesado \\
6 & \\
7 & Muito pesado \\
8 & \\
9 & \\
10 & Máximo \\
\hline
\end{tabular}

\section{Qualidade Total de Recuperação (TQR)}

A escala da TQR de Kenttä \& Hassmén (1998) (quadro 2) é estruturada de acordo com a Escala de Borg (1982) de 6 a 20, no qual 6 significa em nada recuperado e 20 significa totalmente recuperado. Assim, esta escala foi apresentada aos atletas sendo feita a seguinte pergunta: "Como você se sente em relação à sua recuperação?”. 


\section{QUADRO 2}

Escala de Qualidade Total de Recuperação (Kenttã \& Hassmén, 1998).

\begin{tabular}{ll}
\hline 6 & Em nada recuperado \\
7 & Extremamente mal recuperado \\
$\mathbf{8}$ & \\
9 & Muito mal recuperado \\
10 & \\
11 & Mal recuperado \\
12 & \\
13 & Razoavelmente recuperado \\
14 & \\
15 & Bem recuperado \\
16 & \\
17 & Muito bem recuperado \\
18 & \\
19 & Extremamente bem recuperado \\
$\mathbf{2 0}$ & Totalmente bem recuperado \\
\hline
\end{tabular}

Questionário de Bem-Estar (QBE)

Este questionário (quadro 3) avalia cinco domínios: fadiga, qualidade de sono, dor muscular geral, níveis de estresse e de humor. Assim, o atleta avalia em qual nível ele se encontra em uma escala pontuada de 1 a 5, e o bem-estar geral é então determinado pela soma dos cincos escores (McLean et al., 2010). 
QUADRO 3

Questionário de Bem-Estar (McLean et al., 2010)

\begin{tabular}{|c|c|c|c|c|c|c|}
\hline & 5 & 4 & 3 & 2 & 1 & Pontuação \\
\hline Fadiga & $\begin{array}{c}\text { Muito } \\
\text { descansado }\end{array}$ & Descansado & Normal & $\begin{array}{c}\text { Mais cansado } \\
\text { que o normal }\end{array}$ & $\begin{array}{c}\text { Sempre } \\
\text { cansado }\end{array}$ & \\
\hline $\begin{array}{c}\text { Qualidade } \\
\text { de sono }\end{array}$ & $\begin{array}{c}\text { Muito } \\
\text { tranquilo }\end{array}$ & Boa & $\begin{array}{c}\text { Dificuldade } \\
\text { de dormir }\end{array}$ & Sono inquieto & Insônia & \\
\hline $\begin{array}{c}\text { Dor } \\
\text { muscular }\end{array}$ & $\begin{array}{c}\text { Sentindo-se } \\
\text { ótimo }\end{array}$ & $\begin{array}{c}\text { Sentindo-se } \\
\text { bem }\end{array}$ & Normal & Aumento na & $\begin{array}{c}\text { Muito } \\
\text { dolorido }\end{array}$ & \\
\hline $\begin{array}{c}\text { Níveis de } \\
\text { estresse }\end{array}$ & $\begin{array}{c}\text { Muito } \\
\text { relaxado }\end{array}$ & Relaxado & Normal & $\begin{array}{c}\text { Sentindo-se } \\
\text { estressado }\end{array}$ & $\begin{array}{c}\text { Altamente } \\
\text { estressado }\end{array}$ & \\
\hline Humor & $\begin{array}{c}\text { Muito bem } \\
\text { humorado }\end{array}$ & $\begin{array}{c}\text { Em geral } \\
\text { bem } \\
\text { humorado }\end{array}$ & $\begin{array}{c}\text { Menos } \\
\text { interessado } \\
\text { em outras } \\
\text { atividades } \\
\text { que o } \\
\text { normal }\end{array}$ & $\begin{array}{c}\text { "Frieza" com } \\
\text { companheiros } \\
\text { de equipe, } \\
\text { familia e } \\
\text { colegas de } \\
\text { trabalho }\end{array}$ & $\begin{array}{c}\text { Altamente } \\
\text { aborrecido/ } \\
\text { nervoso/ } \\
\text { desanimado }\end{array}$ & \\
& & & & & \\
\hline
\end{tabular}

\section{Análise estatística}

Os resultados são apresentados como média e desvio padrão. Inicialmente, verificou-se a normalidade dos dados através do teste de Shapiro Wilk. Para a homogeneidade das variâncias foi utilizado o teste de Levene. Foi analisada a diferença entre os valores iniciais e finais para cada bloco de semanas, assim como a diferença entre a variação dos scores de TQR e $\mathrm{QBE}(\Delta \mathrm{TQR} / \triangle \mathrm{QBE})$. Atendidos os pressupostos de normalidade, homogeneidade e esfericidade, para comparar os valores da CTST, $\triangle T Q R$ e $\triangle Q B E$ entre as diferentes semanas foi aplicado o teste ANOVA para medidas repetidas com Post-Hock de Bonferroni. Foi ainda calculado o tamanho do efeito ( $\mathrm{d}$ de Cohen) para as mesmas variáveis. Já para a diferença entre os valores de TQR e QBE iniciais e finais para as semanas foi utilizado o teste T pareado. Foi utilizado o software SPSS versão 20.0 e considerado o nível de significância de 5\%.

\section{Resultados E Discuss Ão}

Os valores médios obtidos através da CTST nas semanas sem a realização de jogos foi de 3.813,86 $\pm 1.020,22$, já na semana com um jogo foi 3.583,74 $\pm 592,96$ e com dois jogos o valor foi de 2.921,61 $\pm 709,75$. A semana com 5 jogos apresentou uma CTST de 3.877,72, valor médio maior que as demais situações. No entanto, não foram encontradas diferenças estatisticamente significativas ao comparar as semanas em função da frequência de jogos, como pode ser visto na Tabela 1. Apesar disso, houve um tamanho do efeito muito elevado quando se comparou a diferença entre um e dois jogos semanais, assim como de semanas sem jogos com dois jogos na semana. O tamanho do efeito foi médio ao comparar as semanas sem jogos com as semanas com um jogo. 
TABELA 1

Comparação das semanas levando em conta CTST, $\Delta \mathrm{TQR}$ e $\Delta \mathrm{QBE}$ de acordo com a frequência de jogos.

\begin{tabular}{lcccccc}
\hline Semanas & $\begin{array}{c}\text { CTST } \\
\text { (P valor) }\end{array}$ & $\begin{array}{c}\text { CTST } \\
\text { (TE) }\end{array}$ & $\begin{array}{c}\Delta \text { TQR } \\
\text { (P valor) }\end{array}$ & $\begin{array}{c}\Delta \text { TQR } \\
(\mathrm{TE})\end{array}$ & $\begin{array}{c}\Delta Q B E \\
\text { (P valor) }\end{array}$ & $\begin{array}{c}\Delta \text { QBE } \\
\text { (TE) }\end{array}$ \\
\hline 0 e 1 jogos & 1,000 & 0,285 & 0,063 & 1,183 & $0,003^{*}$ & 1,624 \\
1 e 2 jogos & 0,232 & 1,016 & $0,005^{*}$ & 1,676 & 0,131 & 1,039 \\
0 e 2 jogos & 0,163 & 1,031 & $0,009^{*}$ & 2,629 & $0,001^{*}$ & 3,316 \\
\hline
\end{tabular}

Legenda: $\mathrm{CTST}=$ Carga de Treinamento Semanal Total; $\Delta \mathrm{TQR}=$ Diferença entre a variação dos escores da Qualidade Total de Recuperação; $\Delta \mathrm{QBE}=$ Diferença entre a variação dos escores da Qualidade de bem-estar.

Os valores de variação $\triangle \mathrm{TQR}$ apresentaram diferenças estatísticas entre as semanas com um e dois jogos e para as semanas sem jogos e com dois jogos (Figura 1). Já o $\Delta \mathrm{QBE}$ foi diferente entre semanas sem jogos quando comparado a semanas com um ou dois jogos semanais (Figura 2).

\section{FIGURA 1}

Valores de variação $\triangle \mathrm{TQR}$ de acordo com o número de jogos realizados na semana

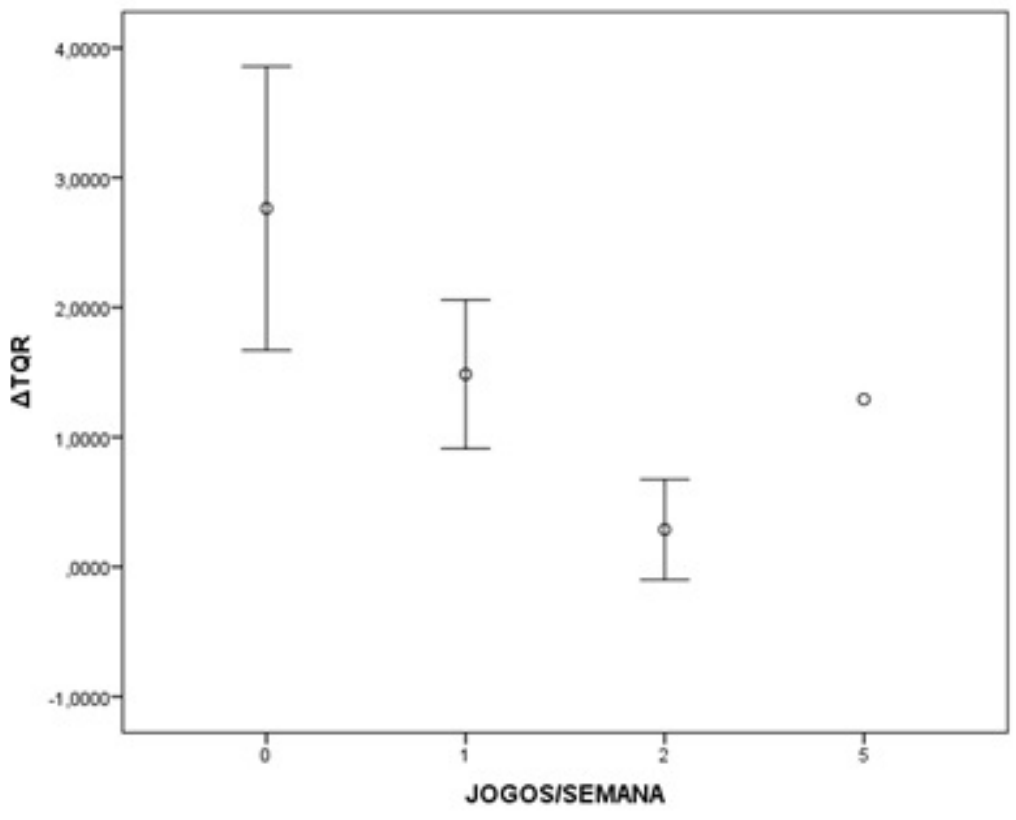


FIGURA 2

Valores de variação $\triangle \mathrm{QBE}$ de acordo com o número de jogos realizados na semana

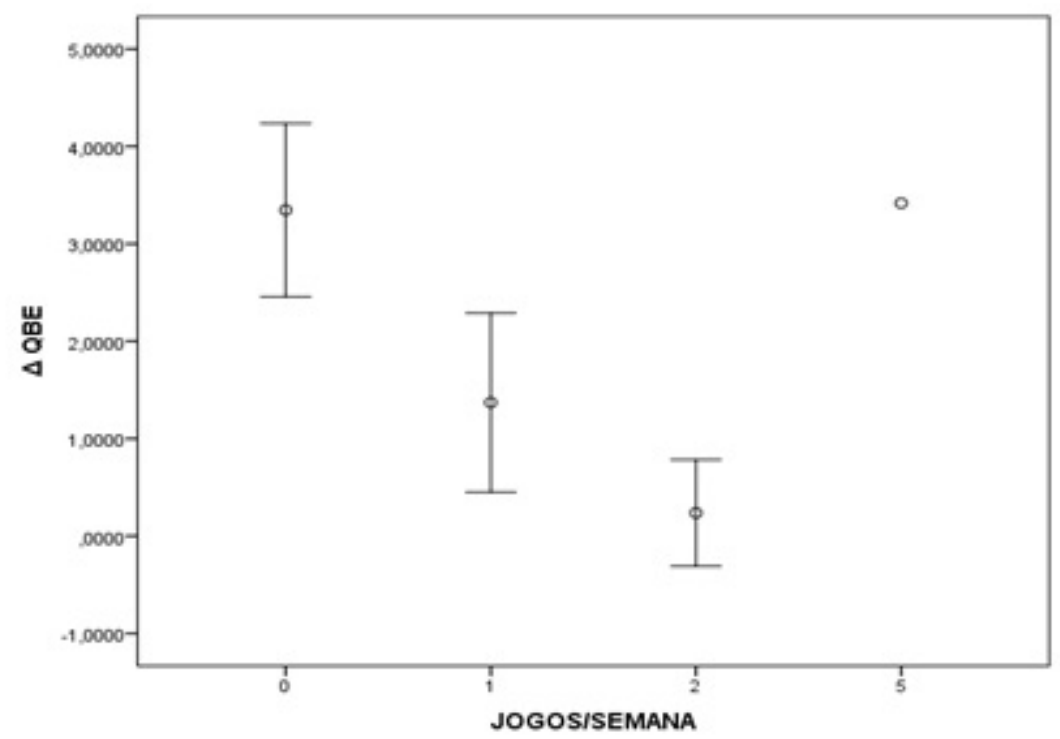

Os escores de TQR inicial podem ser caracterizados dentro da faixa de bem e muito bem recuperados, enquanto a TQR final ficou entre "razoavelmente recuperados" e "bem recuperados”. Já os valores de QBE tendem a uma diminuição quando se compara o último dia de treino com o primeiro. Houve diferença estatisticamente significativa entre a TQR inicial e TQR final para as semanas sem jogos e as semanas com um jogo. Da mesma forma os valores de QBE foram diferentes somente para essas situações (tabela 2).

TABELA 2

Valores iniciais e finais de TQR e QBE de acordo com

o número de jogos realizados na semana (média $\pm \mathrm{DP}$ ).

\begin{tabular}{|c|c|c|c|c|c|c|}
\hline $\begin{array}{l}\text { Jogos/ } \\
\text { Semana }\end{array}$ & TQR inicial & $\begin{array}{l}\text { TQR } \\
\text { final }\end{array}$ & P valor & QBE inicial & $\begin{array}{l}\text { QBE } \\
\text { final }\end{array}$ & P valor \\
\hline 0 & $17,03 \pm 0,39$ & $14,263 \pm 0,94$ & $0,001^{*}$ & $21,90 \pm 0,97$ & $18,55 \pm 0,55$ & $0,000^{*}$ \\
\hline 1 & $17,08 \pm 0,72$ & $15,61 \pm 0,33$ & $0,000^{*}$ & $21,32 \pm 0,92$ & $19.81 \pm 0,89$ & $0,007^{*}$ \\
\hline 2 & $16,06 \pm 0,54$ & $15,58 \pm 0,57$ & 0,129 & $20,04 \pm 0,81$ & $19,78 \pm 0,90$ & 0,358 \\
\hline
\end{tabular}

Legenda: $\mathrm{TQR}=$ Qualidade Total de Recuperação; $\mathrm{QBE}=$ Qualidade de bem-estar; $\mathrm{DP}=$ Desvio Padrão.

Não foram encontradas diferenças estatisticamente significantes entre a CTST em diferentes semanas de acordo com a quantidade de jogos realizados. No entanto, deve-se ressaltar que numericamente estas diferenças são consideráveis dentro do processo de controle da carga de treinamento, fato que não deve ser relevado. Em um artigo semelhante no voleibol, que não fez a distinção quanto ao número de jogos, só apresentou semanas sem e com jogos, também não foram encontradas diferenças significativas (Lacerda et al., 2015). Apesar de não ser encontrada diferenças significativas entre as cargas de treinamento no presente estudo, destaca-se que houve um tamanho de efeito muito elevado entre as semanas com um e dois jogos e as semanas sem jogos quando comparada a semana com dois jogos. Isto é, sob o ponto de vista prático, há uma redução importante das cargas proporcional ao aumento da frequência de jogos. 
Manzi et al. (2010), em um estudo com atletas profissionais de basquete, fizeram essa comparação e não encontraram diferença estatística entre cargas de semanas com um ou dois jogos, porém encontrou diferença entre as semanas sem jogos se comparado aquelas com um ou dois jogos. Este resultado foi semelhante ao presente estudo, no voleibol, demonstrando uma tendência das equipes em reduzir a carga de trabalho em semanas com jogos.

Semanas sem jogos tendem a apresentar cargas maiores se comparados às semanas com jogos uma vez que as cargas podem ser intensificadas já que haverá uma maior disponibilidade de tempo para uma melhor preparação e recuperação dos atletas até a realização da próxima partida. Aliado a isso as cargas dos dias anteriores aos jogos tendem a ser reduzidas. Essa é uma possível explicação para a redução das cargas nas semanas com dois jogos encontradas no presente estudo.

Independentemente do número de jogos na semana e de suas cargas correspondentes, os valores de TQR final encontrados estavam entre "razoavelmente recuperados" e "bem recuperados". Levando-se em consideração as semanas com jogos, a maioria dos jogos aconteceu no final da semana. Entende-se então que os jogadores chegavam para os jogos em um bom estado de recuperação. Valores semelhantes foram encontrados por Lacerda et al. (2015) no qual os atletas também apresentaram valores de TQR final dentro da mesma faixa.

Já os valores de TQR inicial estavam entre "bem recuperados" ou "muito bem recuperados" o que significa que os atletas começaram a semana de treinamentos em boa situação para suportar as cargas de treinamentos e/ou jogos que lhes seriam impostas. Esse comportamento foi observado também independentemente do número de jogos na semana e das cargas correspondentes, sendo encontrados valores semelhantes por Lacerda et al. (2015).

Comparando os valores iniciais e finais de recuperação e bem-estar, observou-se que há uma tendência de queda dos valores finais se comparado aos iniciais. Foram encontradas diferenças estatisticamente significativas para as semanas sem jogos ou com um jogo. $\mathrm{O}$ mesmo não foi encontrado nas semanas com dois jogos em que a TQR e a QBE iniciais e finais foram semelhantes. Como dito anteriormente, não foram encontradas diferenças estatísticas entre as CTST. Porém, um tamanho do efeito elevado para essa variável nas situações: "um e dois jogos" e "sem jogos e dois jogos" pode traduzir que as semanas com dois jogos têm, sob o ponto de vista prático, cargas mais baixas, o que explicaria a pouca variação dos valores de TQR e QBE nessas semanas. Isso vai ao encontro do estudo de Freitas, Nakamura, Miloski, Samulski, \& Bara-Filho (2014) que aponta que cargas de treinamento mais elevadas podem gerar uma diminuição dos valores de recuperação. No estudo de Lacerda et al. (2015) foram encontradas diferenças entre TQR inicial e final se comparado as semanas com e sem jogos, porém o mesmo não fez distinção entre semanas com um ou dois jogos. Johnston et al. (2013) observaram o comportamento da QBE em um período de cinco dias com a realização de três jogos, com intervalo de 48 horas, e encontraram valores menores 12 e 36 horas após a segunda partida se comparado ao score inicial.

Observando a variação da recuperação dos atletas $(\Delta \mathrm{TQR})$ houve decréscimo importante quando havia dois jogos na semana se comparados tanto as semanas sem jogos quanto as semanas com um jogo. Isto significa dizer que dois jogos em uma mesma semana têm potencial efeito sobre a diminuição da recuperação dos atletas no voleibol, o mesmo não ocorrendo quando há a realização de somente uma partida. Lacerda et al. (2015) encontrou diferença nessa variável comparando as semanas sem nenhum jogo ou com jogos. Apesar das variações encontradas, os valores de TQR final estavam dentro de parâmetros aceitáveis para todas as situações.

Quanto aos resultados do $\Delta \mathrm{QBE}$ houve diferença estatística entre as semanas sem a realização de jogos e semanas com jogos, independentemente do número de partidas. Isso pode significar que a realização de jogos tende a diminuir os níveis de bem-estar dos atletas não importando se forem uma ou duas partidas na semana.

Houve uma semana atípica, no qual foi realizado um torneio com 5 jogos. Esta apresentou um valor médio de CTST maior que as demais semanas. Isto pode ser explicado pelo fato de que as cargas dos jogos tendem a 
ser mais altas que a carga dos treinamentos (Henderson, Cook, Kidgell, \& Gastin, 2015). Destaca-se também nessa semana que a $\mathrm{QBE}$ apresentou altos valores de variação, isto pode ser explicado pela influência de fatores como viagem, alta frequência de jogos, e pouco período de recuperação entre as partidas, afetando os escores da QBE, possivelmente em maior proporção nos domínios "sono", "fadiga”, "estresse" e "dor muscular".

\section{Conclusão}

Conclui-se que o período de recuperação (aproximadamente 48 horas entre as semanas) para os atletas de voleibol foi suficiente, já que os valores de TQR inicial estavam entre "bem recuperados" ou "muito bem recuperados", indicando que esses começaram a semana de treinamentos em boa situação para suportar as cargas de treinamentos e/ou jogos que lhes seriam impostas.

Embora não haja uma variação das cargas em diferentes frequências semanais de partidas de voleibol, a recuperação assim como o bem-estar dos atletas varia de acordo com o número de partidas por semana. $\mathrm{Ou}$ seja, quanto maior o número de jogos mais lenta torna-se a recuperação.

Assim, sugere-se que os treinadores e preparadores físicos considerem no planejamento o fato de mesmo com cargas mais baixas, as semanas com jogos geram um estresse psicofisiológico no organismo que afeta diretamente a recuperação dos atletas. Neste sentido, estratégias de periodização e recuperação devem ser implementadas.

\section{REFERÊNCIAS}

Borg, G. A. (1982). Psychophysical bases of perceived exertion. Medicine and Science in Sports and Exercise, 14(5), 377-381. Retrieved from http://www.ncbi.nlm.nih.gov/pubmed/7154893

Borresen, J., \& Lambert, M. I. (2009). The Quantification of Training Load, Effect on Performance. Sports Medicine, 39(9), 779-795. https://doi.org/10.2165/11317780-000000000-00000

Bourdon, P., \& Cardinale, M. (2017). Monitoring Athlete Training Loads: Consensus Statement. Journal of Sports, 12(2), 161-170. https://doi.org/10.1123/IJSPP.2017-0208

Brink, M. S., Nederhof, E., Visscher, C., Schmikli, S. L., \& Lemmink, K. A. P. M. (2010). Monitoring Load, Recovery, and Performance in Young Elite Soccer Players. Journal of Strength and Conditioning Research, 24(3), 597-603. https://doi.org/10.1519/JSC.0b013e3181c4d38b

Foster, C. (1998). Monitoring training in athletes with reference to overtraining syndrome. Medicine and Science in Sports and Exercise, 30(7), 1164-1168. Retrieved from http://www.ncbi.nlm.nih.gov/pubmed/9662690

Foster, C., Florhaug, J. A., Franklin, J., Gottschall, L., Hrovatin, L. A., Parker, S., ... Dodge, C. (2001). A new approach to monitoring exercise training. Journal of Strength and Conditioning Research, 15(1), 109-115. Retrieved from http://www.ncbi.nlm.nih.gov/pubmed/11708692

Freitas, V. H., Nakamura, F. Y., Miloski, B., Samulski, D., \& Bara-Filho, M. G. (2014). Sensitivity of physiological and psychological markers to training load intensification in volleyball players. Journal of Sports Science \& Medicine, 13(3), 571-579. Retrieved from http://www.ncbi.nlm.nih.gov/pubmed/25177184

Govus, A. D., Coutts, A., Duffield, R., Murray, A., \& Fullagar, H. (2018). Relationship Between Pretraining Subjective Wellness Measures, Player Load, and Rating-of-Perceived-Exertion Training Load in American College Football. International Journal of Sports Physiology and Performance, 13(1), 95-101. https://doi.org/10.1123/ ijspp.2016-0714

Henderson, B., Cook, J., Kidgell, D. J., \& Gastin, P. B. (2015). Game and Training Load Differences in Elite Junior Australian Football. Journal of Sports Science \& Medicine, 14(3), 494-500. Retrieved from http:// www.ncbi.nlm.nih.gov/pubmed/26336334 
Impellizzeri, F. M., Rampinini, E., Coutts, A. J., Sassi, A., \& Marcora, S. M. (2004). Use of RPE-based training load in soccer. Medicine and Science in Sports and Exercise, 36(6), 1042-1047. Retrieved from http:// www.ncbi.nlm.nih.gov/pubmed/15179175

Johnston, R. D., Gibson, N. V., Twist, C., Gabbett, T. J., MacNay, S. A., \& MacFarlane, N. G. (2013). Physiological Responses to an Intensified Period of Rugby League Competition. Journal of Strength and Conditioning Research, 27(3), 643-654. https://doi.org/10.1519/JSC.0b013e31825bb469

Kellmann, M. (2010). Preventing overtraining in athletes in high-intensity sports and stress/recovery monitoring. Scandinavian Journal of Medicine \& Science in Sports, 20, 95-102. https://doi.org/10.1111/ j.1600-0838.2010.01192.x

Kenttä, G., \& Hassmén, P. (1998). Overtraining and Recovery. Sports Medicine, 26(1), 1-16. https:// doi.org/10.2165/00007256-199826010-00001

Lacerda, R., Duarte, T., Coimbra, D., Timoteo, T., Marins, J., Miranda, R., \& Bara Filho, M. (2015). Comportamento da recuperação de atletas profissionais de voleibol em semanas com jogos e sem jogos. Coleção Pesquisa Em Educação Física, 14(2), 23-30. Retrieved from http://www.fontouraeditora.com.br/periodico/? $\bmod =$ Arquivo\&opt=Arquivo-Detalhe\&id_arquivo $=1168$

Manzi, V., D\#Ottavio, S., Impellizzeri, F. M., Chaouachi, A., Chamari, K., \& Castagna, C. (2010). Profile of Weekly Training Load in Elite Male Professional Basketball Players. Journal of Strength and Conditioning Research, 24(5), 1399-1406. https://doi.org/10.1519/JSC.0b013e3181d7552a

McLean, B. D., Coutts, A. J., Kelly, V., McGuigan, M. R., \& Cormack, S. J. (2010). Neuromuscular, Endocrine, and Perceptual Fatigue Responses during Different Length Between-Match Microcycles in Professional Rugby League Players. International Journal of Sports Physiology and Performance, 5(3), 367-383. https:// doi.org/10.1123/ijspp.5.3.367

Saw, A. E., Main, L. C., \& Gastin, P. B. (2016). Monitoring the athlete training response: subjective self-reported measures trump commonly used objective measures: a systematic review. British Journal of Sports Medicine, 50(5), 281-291. https://doi.org/10.1136/bjsports-2015-094758

Sheppard, J. M., Gabbett, T. J., \& Stanganelli, L.-C. R. (2009). An Analysis of Playing Positions in Elite Men\#s Volleyball: Considerations for Competition Demands and Physiologic Characteristics. Journal of Strength and Conditioning Research, 23(6), 1858-1866. https://doi.org/10.1519/JSC.0b013e3181b45c6a 